\title{
A Circular Polarizer Designed With a Dielectric Septum Loading
}

\author{
Shih-Wei Wang, Chih-Hung Chien, Chun-Long Wang, and Ruey-Beei Wu, Senior Member, IEEE
}

\begin{abstract}
This paper presents a simple method to realize a circular polarizer by inserting a dielectric septum in the middle of the cylindrical waveguide. Since fixing slots are built to precisely locate the septum, dual-compensation slots are made in the orthogonal plane to balance the effects of the fixing slots. The measured axial ratio is smaller than $0.5 \mathrm{~dB}$ and the phase difference is flat in the frequency range from 60 to $62.25 \mathrm{GHz}$. In addition, the return losses are better than $-13 \mathrm{~dB}$ for both $E_{x}$ and $E_{y}$ polarizations in the same frequency range. This design has the added advantage that poor phase differences resulting from fabrication error or an inaccurate dielectric constant can easily be improved by adjusting the length of the inserted dielectric septum.
\end{abstract}

Index Terms-Circular polarizer, cylindrical waveguide, dielectric septum.

\section{INTRODUCTION}

C IRCULAR polarizers, which play an important role in communication systems, have been widely studied and discussed. One way of realizing a polarizer is to create grooves or irises on the waveguide wall for achieving a $90^{\circ}$ phase difference [1]-[3]. Although these polarizers are robust, precise processes are required when fabricating the grooves and irises. Especially in the $V$-band, it is difficult to modify the polarizer to achieve the required performance if the circuit is malfunctioning. Thus, a polarizer was proposed by placing a metal septum at the center of the waveguide in order to differentiate the phase between the orthogonal modes [4]. Extended research in [5]-[11] discussed the effects of the thickness and shape of the metal septum on the performance of such polarizers. Although such design is simple and effective, it seems that putting a metal septum at the center might introduce large reflection.

Dielectric plates in a waveguide can also introduce circular polarization. An anisotropic dielectric filled waveguide polarizer was proposed as early as 1957 [12]. Since the dielectric constants for $X$ - and $Y$-polarizations are $\varepsilon_{x}$ and $\varepsilon_{y}$, respectively, propagation constants for the two polarizations are different. A further design for minimizing interface reflections was proposed to achieve broad-band characteristics [13]. Another type of polarizer, in which the sidewalls of the waveguide are attached with dielectric slabs, also differentiated the phase between the two orthogonal modes [14]. However, it seems better

Manuscript received August 26, 2003; revised February 15, 2004. This work was supported in part by the Ministry of Education, R.O.C., under Grant 89-E-FA06-2-4, and by the National Science Council under Grant NSC 91-2219-E002-017.

The authors are with the Department of Electrical Engineering and Graduate Institute of Communication Engineering, National Taiwan University, Taipei, Taiwan 10617, R.O.C.

Digital Object Identifier 10.1109/TMTT.2004.830487

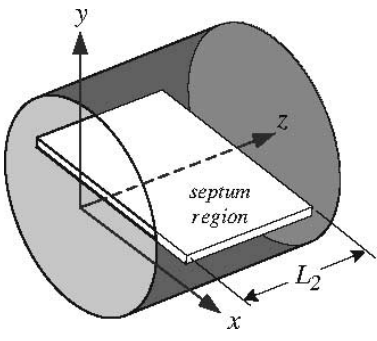

(a)

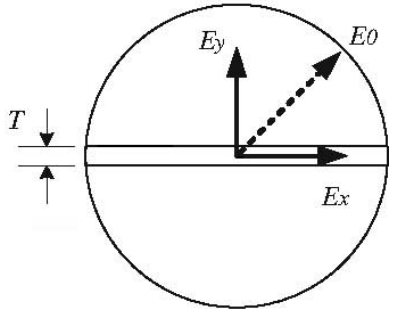

(b)
Fig. 1. Schematic view of the circular polarizer formed with the insertion of dielectric septum. (a) Three-dimensional view. (b) Front view.

if the dielectric slab could be placed at the center of the waveguide where the electric fields are strongest. Accordingly, a dielectric septum in a circular waveguide to construct a polarizer was proposed in [15]. In this paper, a new type of circular polarizer is proposed, which takes advantages of both the metal septum and dielectric sidewall.

\section{Circular Waveguide With Dielectric SePtum OnLy}

\section{A. Description of the Dielectric-Septum Polarizer}

Fig. 1(a) shows a typical circular polarizer, in which a dielectric septum is inserted in the middle of the waveguide. An incident wave $E_{0}$ oriented at $45^{\circ}$ relative to the dielectric septum is assumed, as shown in Fig. 1(b). This wave can be decomposed into two equal orthogonal projections $E_{x}$ and $E_{y}$, respectively parallel and perpendicular to the dielectric septum. The two components then propagate through the septum region with little reflection due to the small septum discontinuity. In the septum region, propagation constant $\beta_{x}$ of the $E_{x}$ component is strongly perturbed by the dielectric septum because the electric field line is parallel to the septum. On the other hand, propagation constant $\beta_{y}$ of the $E_{y}$ component is weakly perturbed because the electric-field line is perpendicular to the septum. As a result, this polarizer can be implemented by choosing a suitable septum length $L_{2}$ so as to realize a $90^{\circ}$ phase difference at the output port.

\section{B. Determination of the Radius of the Circular Waveguide}

The specifications of the polarizer are shown in Table I. Since the polarizer has to operate between the fundamental and the first high-order modes of the waveguide, the radius $R$ needs to fall in the range of $1.58-1.83 \mathrm{~mm}$ to accommodate the operation frequency, which ranges from 60 to $62.25 \mathrm{GHz}$. To design a polarizer, the radius of the circular waveguide must first be determined. The criterion is such that the difference between $\beta_{x}$ 
TABLE I

SPECIFICATIONS OF THE CiRCUlAR POLARIZER

\begin{tabular}{l||c}
\hline Operation band & $60 \sim 62.25 \mathrm{GHz}$ \\
\hline Phase difference & $90^{\circ} \pm 0.5^{\circ}$ \\
\hline Axial ratio & $0.5 \mathrm{~dB}$ \\
\hline
\end{tabular}

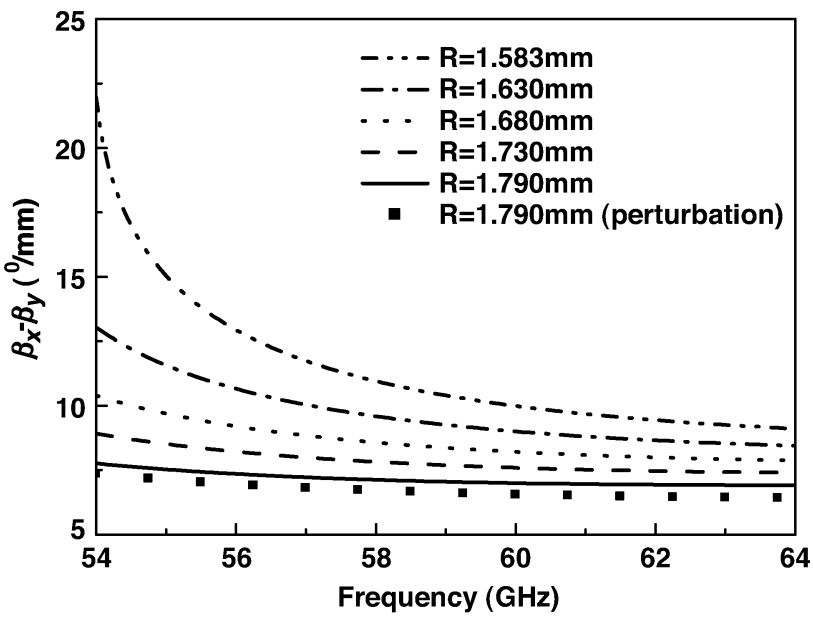

Fig. 2. Difference of propagation constants versus frequency with waveguide radius $R$ as a parameter.

and $\beta_{y}$ in the septum region is frequency insensitive, which then brings out a wide-band response of $90^{\circ}$ phase difference.

Assume then that the dielectric septum is made of Teflon with thickness $T=0.254 \mathrm{~mm}$ and effective dielectric constant 2.2. Fig. 2 shows the simulated results of $\beta_{x}-\beta_{y}$ versus frequency with radius $R$ as a parameter using the eigenmode analysis of the commercial software Ansoft HFSS. It can be seen that a nearly flat response can be obtained with the radius $R=1.79 \mathrm{~mm}$. As can be observed from this figure, the radius $R$ is chosen such that the operation frequency is near the cutoff frequency of the first high-order mode [4]. This figure also shows the results obtained via the perturbation technique [16], which are in close agreement with the simulation results.

\section{Determination of the Length of Dielectric Septum}

According to the above criterion, the radius $R$ of the waveguide is determined to be $1.79 \mathrm{~mm}$. In order to obtain a phase difference of $90^{\circ}$ at $61 \mathrm{GHz}$, the length $L_{2}$ must satisfy

$$
\left(\beta_{x}-\beta_{y}\right) \cdot L_{2}=90^{\circ} \text {. }
$$

With the aid of Fig. 2, the length of the dielectric septum is then calculated to be $12.90 \mathrm{~mm}$.

\section{Consideration of the Effect of Septum Discontinuity}

To address the effect of the septum discontinuity, the full structure of Fig. 1 is simulated via Ansoft HFSS. Fig. 3 shows the phase difference versus frequency with septum length $L_{2}$ as a parameter. As can be seen from this figure, the full-wave simulation result deviated less from the two-dimensional solution for the case corresponding to $L_{2}=12.90 \mathrm{~mm}$. This indicates

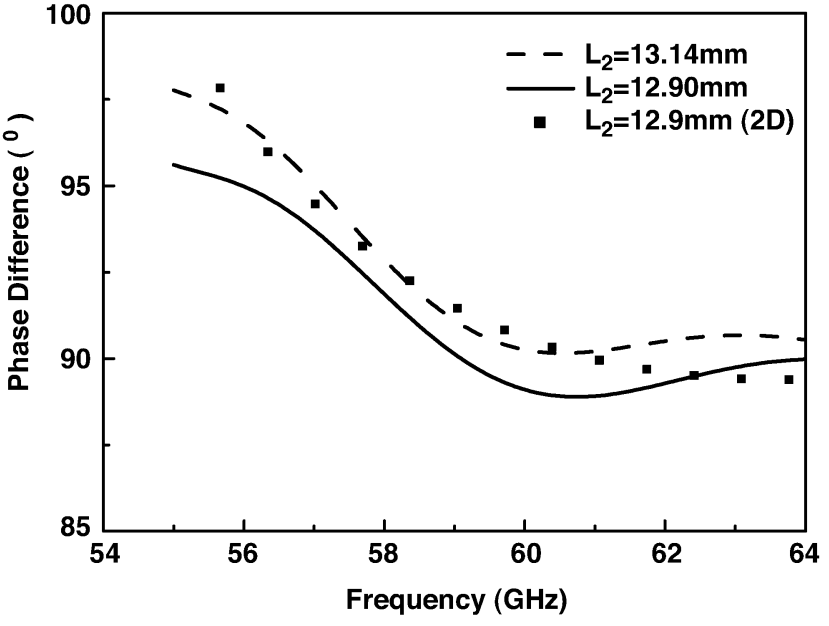

Fig. 3. Phase difference versus frequency with septum length $L_{2}$ as a parameter.

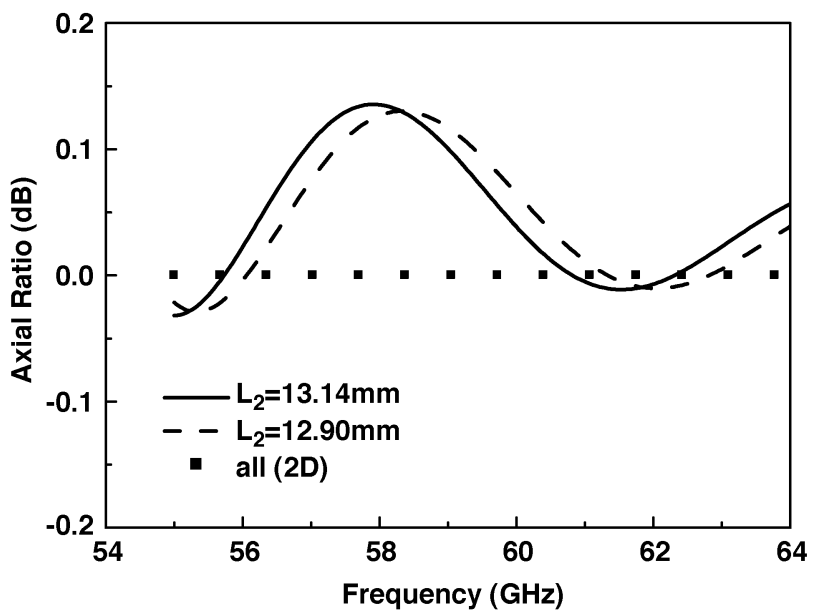

Fig. 4. Axial ratio versus frequency with septum lengths $L_{2}$ as a parameter.

that the septum discontinuity has little effect on the phase difference. In order to maintain a nearly $90^{\circ}$ phase difference in the frequency range from 60 to $62.25 \mathrm{GHz}, L_{2}$ is increased to a value of $13.14 \mathrm{~mm}$.

Fig. 4 shows the results of the axial ratio $\left(E_{x}\right.$ over $\left.E_{y}\right)$ versus frequency for different values of the septum length $L_{2}$. It can be seen that the axial ratio was only slightly disturbed by the change in septum length. Moreover, the results of the full-wave simulation deviated slightly from $0 \mathrm{~dB}$ corresponding to the two-dimensional solution, which means that the septum discontinuity also has a small effect on the axial ratio. The return loss versus frequency for the two polarizations $E_{x}$ and $E_{y}$ are shown in Fig. 5. It can be seen that return losses are better than $-22 \mathrm{~dB}$ for $E_{x}$ polarization and $-16 \mathrm{~dB}$ for $E_{y}$ polarization in the frequency range from 60 to $62.25 \mathrm{GHz}$.

\section{Circular Waveguide With Dielectric SEPTUM AND SLOTS}

From a practical point-of-view, slots are required in the polarizer to precisely locate the dielectric septum despite having a negative impact on polarizer performance. To balance the effect of the fixing slots, compensation slots are also made in the 


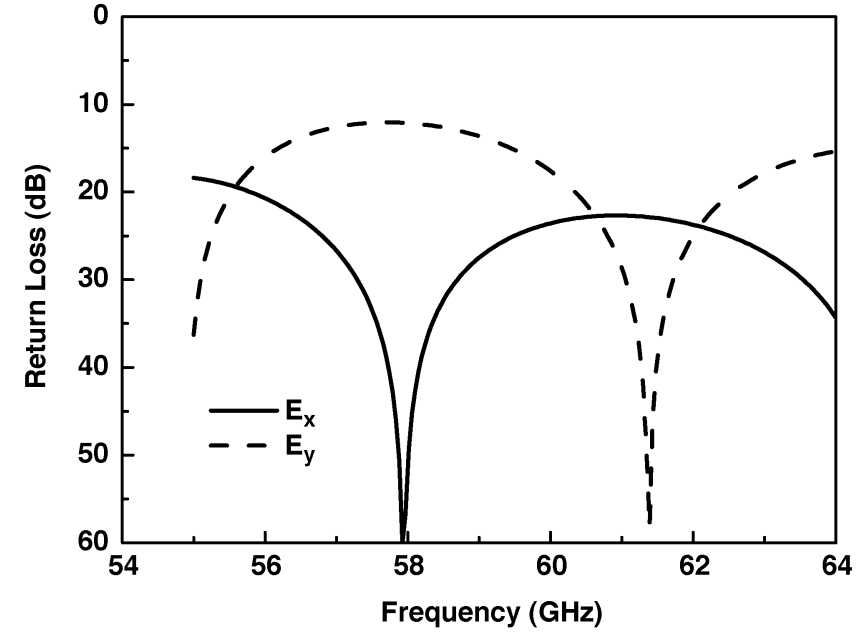

Fig. 5. Return loss versus frequency for $x$ and $y$ polarizations with $L_{2}=$ $13.14 \mathrm{~mm}$.

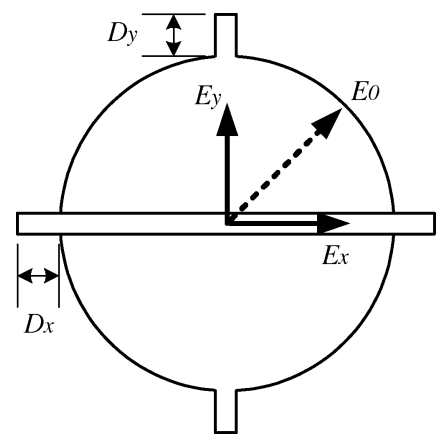

Fig. 6. Cross-sectional view of a polarizer with slots in the septum region.

$y$-direction of the circular polarizer, as shown in Fig. 6. After calculating the propagation constants for the circular polarizer, it was found that the flatness of $\beta_{x}-\beta_{y}$ is maintained. Based on this result, the length of the dielectric septum was calculated to be $18.5 \mathrm{~mm}$.

To investigate the influence of the septum discontinuity, the full structure was simulated using Ansoft HFSS. The polarizer was also fabricated and measured to verify the simulation results. The measurements were obtained using HP8510C after calibrating by the waveguide standard kits, and then adding rectangular-to-circular waveguide transition. The $E_{x}$ and $E_{y}$ polarizations were measured separately to determine their $S$-parameters. The phase difference was calculated from the phases of the two $S_{12}$-parameters, while the axial ratio was found from the division of their magnitudes.

Fig. 7 compares the simulation and measurement results for phase difference versus frequency. It can be seen that the phase difference simulated via Ansoft HFSS fell within $90.4^{\circ} \sim 91.72^{\circ}$ in the frequency range from 60 to $62.25 \mathrm{GHz}$. This is in close agreement with those expected from the two-dimensional results, but deviates significantly from the measurement data. This discrepancy between measurement and simulation might be attributed to fabrication error or an incorrect dielectric constant in the $V$-band. In light of the linear relationship between the phase difference and septum length, this discrepancy can be easily corrected by proportionally

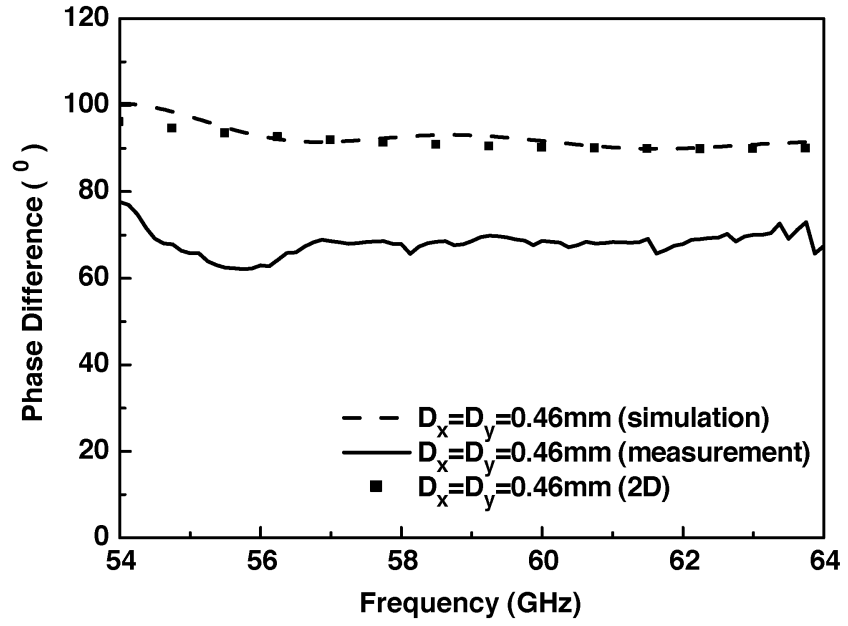

Fig. 7. Phase difference versus frequency with slot depth $D_{x}=D_{y}=$ $0.46 \mathrm{~mm}$.

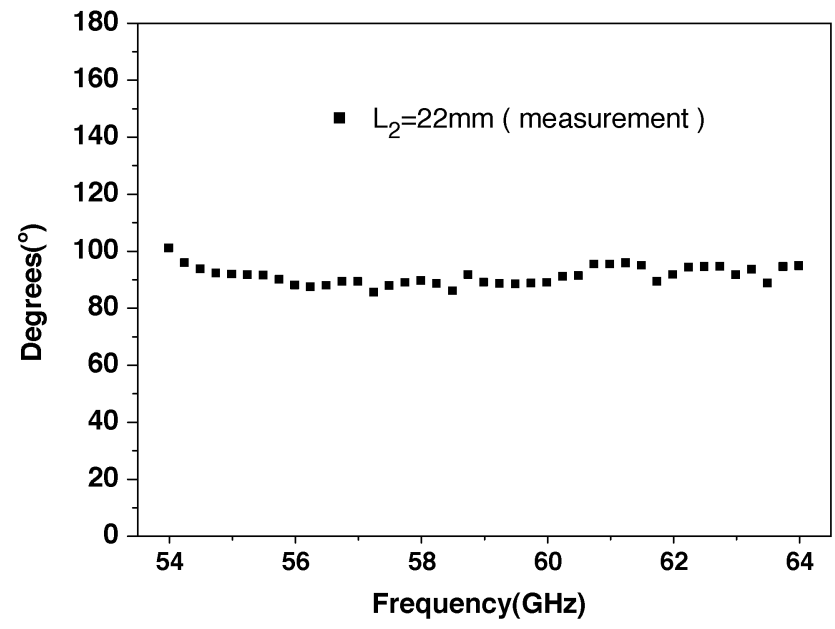

Fig. 8. Phase difference with $L_{2}=22 \mathrm{~mm}$.

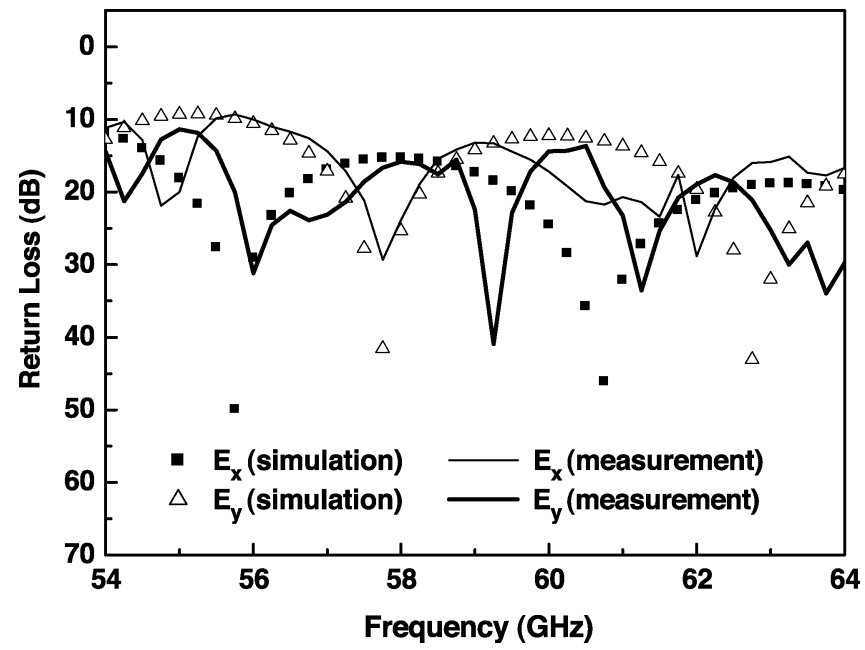

Fig. 9. Return loss for $x$ - and $y$-polarizations.

adopting a longer dielectric septum $L_{2}$. We changed the septum length to $L_{2}=22 \mathrm{~mm}$ and discovered that the phase difference approached $90^{\circ}$, as is evident from Fig. 8. 


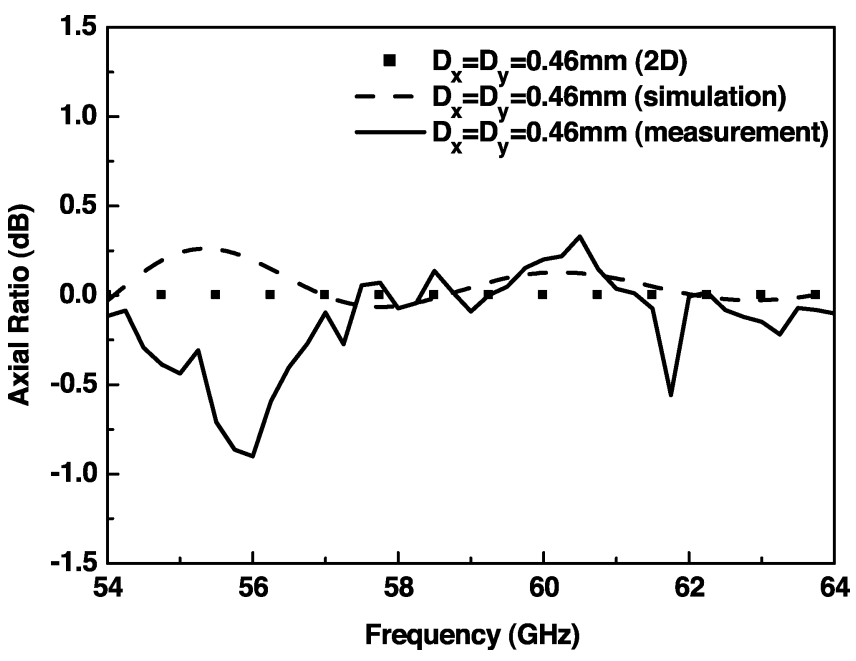

Fig. 10. Axial ratio versus frequency.

The return loss versus frequency for the two polarizations $E_{x}$ and $E_{y}$ is shown in Fig. 9. It can be seen that the measured return losses are better than $-13 \mathrm{~dB}$ for both $E_{x}$ and $E_{y}$ polarizations in the frequency range from 60 to $62.25 \mathrm{GHz}$. Axial ratio versus frequency is shown in Fig. 10 and the measured data are smaller than $\pm 0.5 \mathrm{~dB}$ in the frequency range of interest.

\section{CONCLUSIONS}

A new type of circular polarizer using the simple insertion of a dielectric septum has been proposed in this paper. The resulting axial ratio is smaller than $0.5 \mathrm{~dB}$ and the phase difference is flat and close to $90^{\circ}$ in the frequency range from 60 to $62.25 \mathrm{GHz}$. The return losses are better than $-13 \mathrm{~dB}$ for both $E_{x}$ and $E_{y}$ polarizations in the same frequency range. Due to the structural symmetry of the polarizer, its performance can be easily improved by adjusting the length of the inserted dielectric septum when the phase difference does not meet desired specifications.

\section{ACKNOWLEDGMENT}

The authors wish to acknowledge AirWave Inc., Taipei, Taiwan, R.O.C., for fabricating the polarizer circuit for this study.

\section{REFERENCES}

[1] N. Yoneda, M. Miyazaki, H. Matsumura, and M. Yamato, "A design of novel grooved circular waveguide polarizers," IEEE Trans. Microwave Theory Tech., vol. 48, pp. 2446-2452, Dec. 2000.

[2] N. Yoneda, M. Miyazaki, T. Horie, and H. Satou, "Mono-grooved circular waveguide polarizers," in IEEE MTT-S Int. Microwave Symp. Dig., 2002, pp. 821-824.

[3] G. Bertin, B. Piovano, L. Accatino, and M. Mongiardo, "Full-wave design and optimization of circular waveguide polarizers with elliptical irises," IEEE Trans. Microwave Theory Tech., vol. 50, pp. 1077-1083, Apr. 2002.

[4] M. H. Chen and G. N. Tsandoulas, "A wide-band square-waveguide array polarizer," IEEE Trans. Antennas Propagat., vol. AP-31, pp. 389-391, May 1973.

[5] N. C. Albertsen and P. Skov-Madsen, "A compact septum polarizer," IEEE Trans. Microwave Theory Tech., vol. MTT-31, pp. 654-660, Aug. 1983.
[6] T. Ege and P. McAndrew, "Analysis of stepped septum polarizers," Electron. Lett., vol. 21, no. 24, pp. 1166-1168, Nov. 1985.

[7] R. Behe and P. Brachat, "Compact duplexer-polarizer with semicircular waveguide," IEEE Trans. Antennas Propagat., vol. 39, pp. 1222-1224, Aug. 1991.

[8] J. Esteban and J. M. Rebollar, "Field theory CAD of septum OMT-polarizers," in IEEE AP-S Symp. Dig., 1992, pp. 2146-2149.

[9] R. Ihmels, U. Papziner, and F. Arndt, "Field theory design of a corrugated septum OMT," in IEEE MTT-S Int. Microwave Symp. Dig., 1993, pp. 909-911.

[10] J. Bornemann and V. A. Labay, "Ridge waveguide polarizer with finite and stepped-thickness septum," IEEE Trans. Microwave Theory Tech., vol. MTT-43, pp. 1782-1787, Aug. 1995.

[11] J. Bornemann and S. Amari, "Septum polarizer design for antenna feeds produced by casting," in IEEE AP-S Symp. Dig., 1997, pp. 1422-1425.

[12] H. S. Kirschbaum and S. Chen, "A method of producing broad-band circular polarization employing an anisotropic dielectric," IEEE Trans. Microwave Theory Tech., vol. MTT-5, pp. 199-203, July 1957.

[13] E. M. Kennaugh and S. Adachi, "The analysis of a broad-band circular polarizer including interface reflections," IEEE Trans. Microwave Theory Tech., vol. MTT-8, pp. 520-525, Sept. 1960.

[14] E. Lier and T. Schaug-Pettersen, "A novel type of waveguide polarizer with large cross-polar bandwidth," IEEE Trans. Microwave Theory Tech., vol. 36, pp. 1531-1534, Nov. 1988.

[15] W. D. Cornelius, "Waveguide assembly and circular polarizer for 2450 MHz ECR ion source," in Particle Accelerator Conf., vol. 3, Mar.-Apr. 1999, pp. 1887-1889.

[16] R. F. Harrington, Time-Harmonic Electromagnetic Fields. New York: McGraw-Hill, 1961, ch. 7

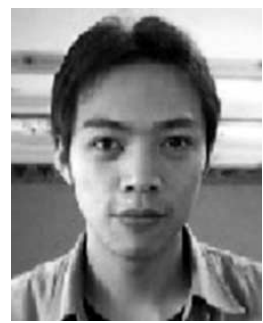

Shih-Wei Wang was born in Taipei, Taiwan, R.O.C, in 1978. He received the B.S. degree in communication engineering from the National Chiao-Tung University, Hsinchu, Taiwan, R.O.C., in 2001, and the M.S. degree in electrical engineering from the National Taiwan University, Taipei, Taiwan, R.O.C., in 2003.

His research interests include microwave and millimeter-wave planar filters and waveguide polarizer.

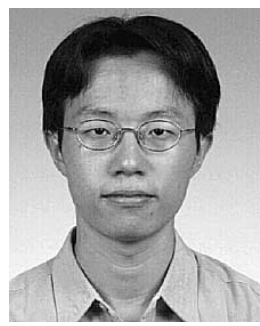

Chih-Hung Chien was born in Taipei, Taiwan, R.O.C, in 1980. He received the B.S. degree in communication engineering from the National Chiao-Tung University, Hsinchu, Taiwan, R.O.C., in 2002, and is currently working toward the M.S degree in electrical engineering at the National Taiwan University, Taipei, Taiwan, R.O.C.

His research interests include microwave and millimeter-wave planar filters and planar circuits to waveguide transitions.

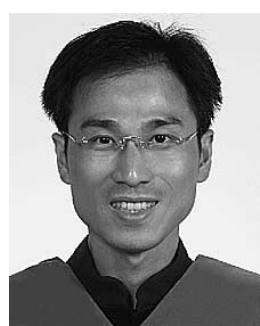

Chun-Long Wang was born in Taichung, Taiwan, R.O.C., in 1972. He received the B.S. and M.S. degrees in communication engineering from the National Chiao-Tung University, Hsinchu, Taiwan, R.O.C., in 1995 and 1997, respectively, and the $\mathrm{Ph} . \mathrm{D}$. degree in communication engineering from the National Taiwan University, Taipei, Taiwan, R.O.C., in 2003.

His areas of interest include flip-chip transition and filter designs. 


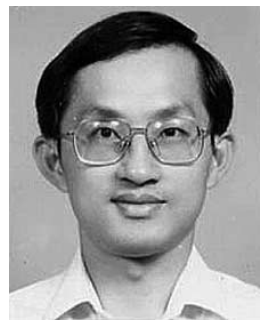

Ruey-Beei Wu (M'91-SM'97) received the B.S.E.E. and $\mathrm{Ph} . \mathrm{D}$. degrees from the National Taiwan University, Taipei, Taiwan, R.O.C., in 1979 and 1985, respectively.

He then joined the faculty of the Department of Electrical Engineering, National Taiwan University, where he is currently a Professor. He is also with the Graduate Institute of Communications Engineering, which was established in 1997. From March 1986 to February 1987, he was a Visiting Scholar with IBM, East Fishkill, NY. From August 1994 to July 1995, he was with the Electrical Engineering Department, University of California at Los Angeles. From May 1998 to April 2000, he became Director of the National Center for High-performance Computing. Since November 2002, he has also served as Director of the Planning and Evaluation Division, National Science Council. His areas of interest include computational electromagnetics, transmission-line and waveguide discontinuities, microwave and millimeter-wave planar circuits, and interconnection modeling for computer packaging. He has authored or coauthored over 50 publications appearing in IEEE publications and over 70 papers appearing in international conferences.

Dr. Wu is a member of the Phi Tau Phi Scholastic Society, the Chinese Institute of Engineers, the Chinese Institute of Electrical Engineers, and the International Union of Radio Science (URSI) 